OPEN ACCESS

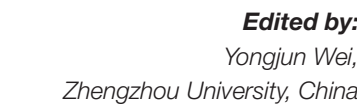

Reviewed by: Jie Sun,

Zhejiang University of Technology, China

Kentaro Inokuma Kobe University, Japan

${ }^{*}$ Correspondence:

Xia Wu

wuxia@sust.edu.cn Jian Zha

zhajian1985@sust.edu.cn; zhaj1985@126.com

Specialty section:

This article was submitted to Microbial Physiology and Metabolism, a section of the journal

Frontiers in Microbiology

Received: 29 November 2020

Accepted: 11 January 2021

Published: 03 February 2021

Citation:

Zha J, Yuwen M, Qian W and

Wu X (2021) Yeast-Based Biosynthesis of Natural Products

From Xylose.

Front. Bioeng. Biotechnol. 9:634919. doi: 10.3389/fbioe.2021.634919

\section{Yeast-Based Biosynthesis of Natural Products From Xylose}

\author{
Jian Zha*, Miaomiao Yuwen, Weidong Qian and Xia Wu* \\ School of Food and Biological Engineering, Shaanxi University of Science and Technology, Xi'an, China
}

Xylose is the second most abundant sugar in lignocellulosic hydrolysates. Transformation of xylose into valuable chemicals, such as plant natural products, is a feasible and sustainable route to industrializing biorefinery of biomass materials. Yeast strains, including Saccharomyces cerevisiae, Scheffersomyces stipitis, and Yarrowia lipolytica, display some paramount advantages in expressing heterologous enzymes and pathways from various sources and have been engineered extensively to produce natural products. In this review, we summarize the advances in the development of metabolically engineered yeasts to produce natural products from xylose, including aromatics, terpenoids, and flavonoids. The state-of-the-art metabolic engineering strategies and representative examples are reviewed. Future challenges and perspectives are also discussed on yeast engineering for commercial production of natural products using xylose as feedstocks.

Keywords: xylose, yeast, natural product, Saccharomyces cerevisiae, Pichia stipitis, Yarrowia lipolytica

\section{INTRODUCTION}

Biomass hydrolysates are frequently used as a feedstock in biomass-based biorefinery. The conversion of these hydrolysates to useful compounds, such as natural products, is usually carried out by yeasts due to their advantageous properties such as tolerance of toxic inhibitors in hydrolysates, functional expression of eukaryote-derived heterologous pathways, and resistance to osmotic stress and harmful fermentation stimuli (Kwak et al., 2019; Li et al., 2019). A major challenge in this process is a waste of resources and high process cost partly due to inefficient utilization of xylose, which is the second most abundant saccharide in biomass hydrolysates (Zha et al., 2012, 2014). Xylose cannot be naturally metabolized by the commonly used yeast chassis such as Saccharomyces cerevisiae and Yarrowia lipolytica with the exception of Scheffersomyces stipitis (Jagtap and Rao, 2018). To overcome this difficulty, there have been many attempts over the past few decades on yeast engineering for xylose fermentation, with Sa. cerevisiae as the main focus (Hou et al., 2017).

The early endeavor of yeast engineering largely aimed at bioethanol production using $S a$. cerevisiae and Sc. stipitis due to their intrinsic capability of synthesizing ethanol from various carbon sources. However, the complicated production process, including pretreatment, saccharification, and fermentation, results in high production cost and limited market competitiveness, and the relevant technologies are far from industrialization on a large scale (Kwak et al., 2019). In consequence, researchers started to explore yeast-based conversion of xylose, together with glucose, into value-added chemicals that are not readily available through extraction or chemical synthesis (Kwak and Jin, 2017; Kwak et al., 2019). 
Although glucose is a preferred carbon source for many microbes, it is not always better than xylose when particular metabolic requirements need to be met. Xylose can induce respiratory effects on central carbon metabolism even under anaerobic conditions, and the metabolic flux of the TCA cycle, the pentose phosphate pathway (PPP), and acetyl-CoA biosynthesis is much higher on xylose than on glucose (Kwak et al., 2019). Compared with glucose, xylose induces a different metabolic flux distribution and enhances the generation of some key intermediate metabolites such as acetyl-CoA, malonyl-CoA, and erythrose-4-phosphate (E-4-P; Kwak and Jin, 2017). The elevated supply of these precursors is beneficial for the production of several classes of natural compounds such as terpenoids and aromatics.

In this review, we will summarize the recent progress on genetic modification of yeast strains for the biosynthesis of natural products using xylose as the carbon source, with a focus on three yeasts including the natural xylose-fermenting yeast Sc. stipitis and recombinant xylose-fermenting yeasts $\mathrm{Sa}$. cerevisiae and Y. lipolytica. For each of these organisms, the description will be mainly centered on four aspects, which include general physiology related to metabolic properties, tools, and strategies available for genetic manipulation, metabolism of xylose, and the biosynthesis of typical natural products from xylose.

\section{XYLOSE METABOLIC PATHWAYS}

Three xylose catabolic pathways have been discovered so far in natural xylose-utilizing microorganisms (Figure 1). The first pathway is the XR-XDH pathway widely present in natural xylose-utilizing yeasts, such as Sc. stipitis and Candida shehatae,

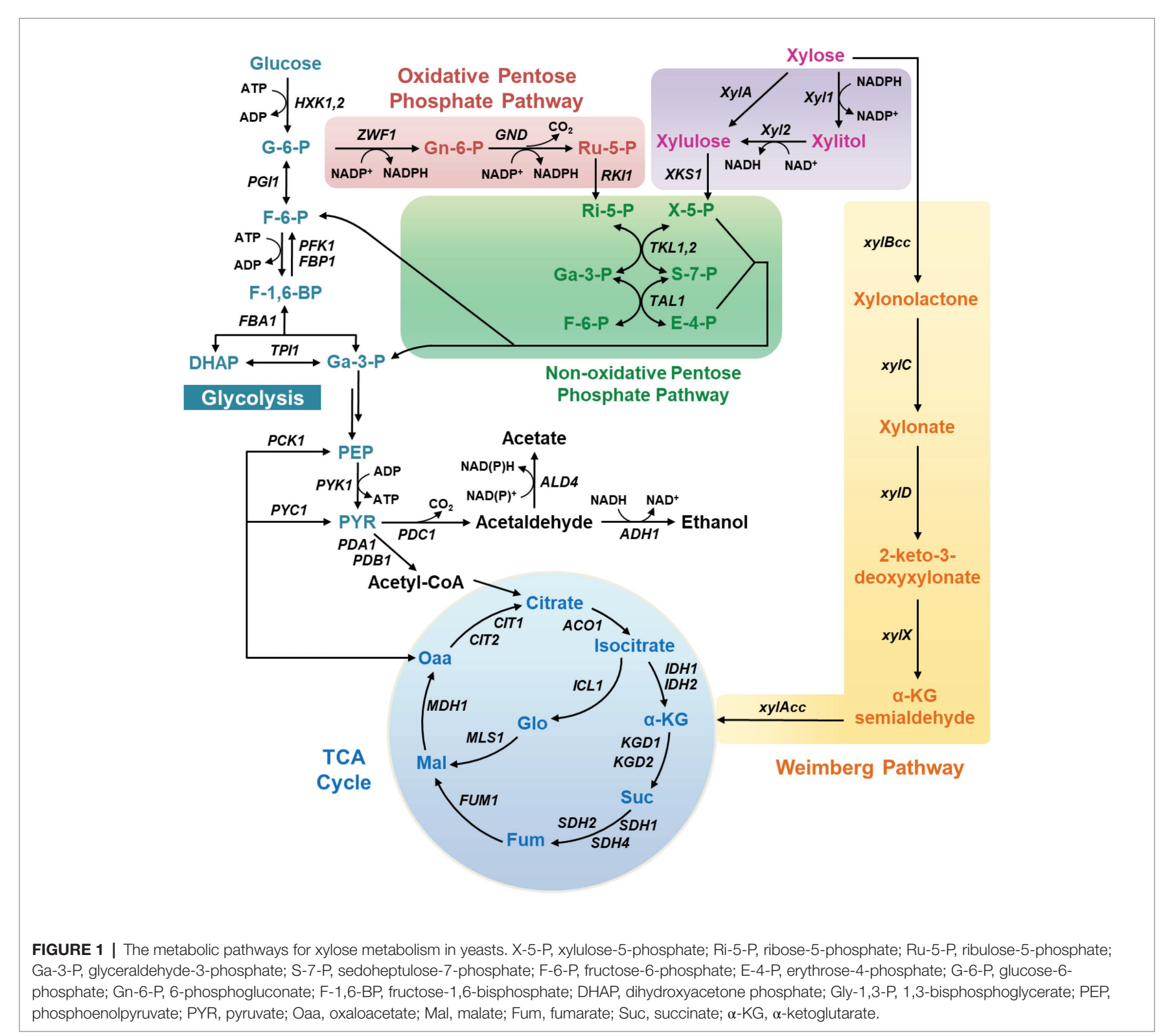


in which xylose is reduced to xylitol by xylose reductase (XR) and then oxidized to xylulose by xylitol dehydrogenase $(\mathrm{XDH}$; Figure 1).

A more direct pathway is the XI pathway that converts xylose into xylulose in a single step using xylose isomerase (XI; Figure 1), after which xylulose can be channeled into glycolysis via phosphorylation and multiple biochemical reactions in the non-oxidative PPP. The XI pathway is intrinsic in bacteria and a few fungi.

The third xylose metabolic pathway is the Weimberg pathway, in which xylose is oxidized by xylose dehydrogenase (XylB) to xylono- $\gamma$-lactone and converted to xylonate by xylono- $\gamma$ lactone lactonase (XylC; Figure 1). Xylonate then undergoes two successive dehydration reactions by xylonate dehydratase (XylD) and 2-keto-3-deoxy-xylonate dehydratase (XylX) to form $\alpha$-ketoglutarate semialdehyde, which is further oxidized to $\alpha$-ketoglutarate by $\alpha$-ketoglutarate semialdehyde dehydrogenase (XylA) and enters the TCA cycle.

Among these pathways, the $\mathrm{XR}-\mathrm{XDH}$ route is the mostly explored in recombinant yeasts owing to its ease of expression and high metabolic flux, and this route has been successfully expressed in non-xylose-fermenting yeasts including Sa. cerevisiae and Y. lipolytica (Kim et al., 2013; Wu et al., 2019). The functional expression of bacterial XI pathway in non-xylosefermenting yeasts is a challenge, whereas the XI pathway derived from Piromyces and other organisms operates well in Sa. cerevisiae (Kuyper et al., 2003). In comparison, the introduction of the Weimberg pathway is much more challenging, which has only been achieved recently in Sa. cerevisiae with albeit low efficiency of xylose metabolism (Borgström et al., 2019). In this work, the gene $x y l A$ was replaced with its ortholog $K s a D$ from Corynebacterium glutamicum, and gene expression in the lower Weimberg pathway ( $X y l D, X y l X$, and $K s a D)$ was enhanced. Through further deletion of the iron regulon repressor FRA2 and serial adaptive evolution, the engineered strain was capable of metabolizing up to $57 \%$ of the carbon from assimilated xylose into biomass and carbon dioxide in the mixture of glucose and xylose. The growth using xylose as the sole carbon source has not been reported, possibly due to the weak activity of the introduced Weimberg pathway.

\section{BIOSYNTHESIS OF NATURAL PRODUCTS FROM XYLOSE BY SCHEFFERSOMYCES STIPITIS}

Scheffersomyces stipitis, previously known as Pichia stipitis, is a facultatively anaerobic yeast that exists mostly in haploid form (Jeffries et al., 2007). This microbe can metabolize many lignocellulose-derived saccharides including hexoses, pentoses, and cellobiose attributed to various hydrolases encoded by its genome such as $\beta$-glucosidases, endoglucanases, xylanase, mannanase, and chitinase. Such a high diversity of carbon sources for this yeast may be a result of long adaptation to its natural habitat, as several known Sc. stipitis strains, such as CBS 6054, naturally dwell in insects that feed on lignocellulose. This unique capability makes Sc. stipitis, a promising strain to utilize lignocellulosic biomass as feedstocks, to produce biofuels and chemicals (Gao et al., 2017).

Scheffersomyces stipitis is a Crabtree negative yeast with greater respiratory capacity than $\mathrm{Sa}$. cerevisiae due to the presence of an alternative respiration system beyond cytochrome system that is sensitive to salicylhydroxamic acid, the so-called SHAMsensitive respiratory pathway (Jeppsson et al., 1995; Jeffries et al., 2007). This pathway branches out of the cytochrome pathway at ubiquinone, donating electrons directly to $\mathrm{O}_{2}$ to form water (Jeppsson et al., 1995). Scheffersomyces stipitis cells growing without a functional cytochrome pathway can still metabolize xylose although the growth rate is reduced by half (Shi et al., 1999). In addition, this yeast contains NADH dehydrogenase complex I, which is absent in Sa. cerevisiae, for ATP generation through oxidative phosphorylation (Jeffries et al., 2007). Scheffersomyces stipitis is more stable than Sa. cerevisiae in terms of metabolite accumulation in response to oxygen supply, and its metabolic flux distribution is greatly affected by oxygen levels, which is different from Sa. cerevisiae that significantly relies on glucose concentrations for metabolic regulation.

Scheffersomyces stipitis is a natural xylose utilizer that harbors the XR/XDH pathway. XR uses either NADPH or NADH as cofactor, and excess $\mathrm{NADH}$ is produced when cells are grown on xylose, allowing for oxygen-independent utilization of xylose (Bruinenberg et al., 1984; Does and Bisson, 1989; Jeffries et al., 2007). The Sc. stipitis genome encodes multiple enzymes to participate in $\mathrm{NAD}(\mathrm{P}) \mathrm{H}$ oxidoreductase reactions for balancing of cofactors and redox potential (Jeffries et al., 2007). This organism has the highest native capability of xylose fermentation among all the known microbes, and its rate of xylose uptake and cell growth, when xylose is used as the sole carbon source, is one order of magnitude higher than that of the recombinant $\mathrm{Sa}$. cerevisiae harboring the $\mathrm{XR} / \mathrm{XDH}$ pathway (Jeffries et al., 2007; Feng and Zhao, 2013). In Sc. stipitis, xylose uptake is a rate-limiting step in xylose metabolism under aerobic conditions, whereas xylulose formation from xylitol is ratelimiting under anaerobic conditions (Ligthelm et al., 1988). There are two types of xylose transporters termed the highand low-affinity transport systems, both of which are proton symports and can be inhibited by dinitrophenol, indicating active transport (Does and Bisson, 1989). The uptake of xylose can be inhibited by glucose competitively and non-competitively in the low-affinity and high-affinity transport systems, respectively (Kilian and van Uden, 1988; Jeffries et al., 2007).

Scheffersomyces stipitis uses CUG to encode serine instead of leucine, which makes codon optimization extremely important when heterologous genes contain leucine-encoding CTG. In addition, nonhomologous end joining (NHEJ) dominates DNA repair of double strand breaks, leading to very low efficiency $(<10 \%)$ of genome editing (Cao et al., 2018). NHEJ in this organism relies on the dimeric protein complex $\mathrm{Ku}$ consisting of Ku70 and Ku80 subunits that binds to ends of DNA double strand breaks. Deletion of the Ku complex increases the efficiency of homologous recombination to $\sim 70 \%$. Using such a $\mathrm{Ku}^{-}$ background, CRISPR-Cas9 and CRISPR-dCas9 systems have been developed in Sc. stipitis, which has greatly facilitated the basic genetic engineering. Moreover, a wide spectrum of native 
promoters and terminators has been reported, and a centromeric DNA sequence has been identified to stabilize episomal plasmids for stable expression of heterologous genes (Cao et al., 2017). These findings and tools make genetic engineering precise and facile in Sc. stipitis.

So far, Sc. stipitis is not as a popular host as Sa. cerevisiae in the production of natural products. This is partly due to the limited genetic tools such as expression plasmids and gene knock-out tools. On the other hand, the physiology and genetics of Sc. stipitis are less defined compared with Sa. cerevisiae. Nevertheless, Sc. stipitis has high metabolic flux toward PPP and abundant supply of E-4-P (Jeffries et al., 2007), which is the precursor of aromatics, therefore, this yeast is suitable for the biosynthesis of aromatics (Gao et al., 2017; Cao et al., 2018). Recently, Sc. stipitis was used to synthesize shikimate from xylose (Gao et al., 2017). In this strain, overexpression was performed on $\operatorname{aro}_{\mathrm{K} 220 \mathrm{~L}}, t k t 1$, and $\operatorname{aro} 1_{\mathrm{D} 900 \mathrm{~A}}$, which encode a feedback insensitive DAHP synthase, a transketolase, and a pentafunctional enzyme converting DAHP to 5-enolpyruvyl3-shikimate phosphate, respectively. Additionally, promoters and terminators were optimized to ensure strong constitutive expression of the pathway genes. These strategies allowed for the production of $3.11 \mathrm{~g} / \mathrm{L}$ shikimate from xylose under aerobic conditions, which was 7-fold higher than the highest reported titer ever achieved in Sa. cerevisiae. This work opens the door to the biosynthesis of aromatic compounds in Sc. stipitis.

\section{USING SACCHAROMYCES CEREVISIAE AS THE HOST TO PRODUCE NATURAL PRODUCTS FROM XYLOSE}

Saccharomyces cerevisiae is one of the mostly used model hosts in metabolic engineering. It is a single-celled GRAS (generally regarded as safe) fungus that proliferates through budding or fission. Saccharomyces cerevisiae has high tolerance to environmental stress, such as low $\mathrm{pH}$, high osmotic pressure, and phage infection, making it advantageous in industrial fermentation. Moreover, the well-defined physiological information and sophisticated metabolic engineering tools of this yeast greatly facilitate its engineering and applications.

Natural Sa. cerevisiae contains native xylose metabolic pathway genes, such as aldose reductase-encoding GRE3, sorbitol dehydrogenase-encoding SOR1 and xylulose kinase (XKS) gene $X K S 1$, the counterpart of XYL1, XYL2, and XYL3 from Sc. stipitis (Konishi et al., 2015). However, these genes are not expressed sufficiently to support significant growth on xylose. Metabolic engineering efforts have created excellent xyloseutilizing recombinant $S$ a cerevisiae through various approaches including the introduction of efficient heterologous xylose metabolic pathway, activation of endogenous genes involved in xylose utilization, enhancement of the PPP, balance of cofactors, evolutionary engineering using xylose as the sole carbon source, and so on (Hou et al., 2017). These attempts have potentiated $\mathrm{Sa}$. cerevisiae as a robust chassis organism in generating a wide variety of useful compounds using xylose alone or together with glucose as the carbon source.
Unlike Sc. stipitis, Sa. cerevisiae is a Crabtree-positive strain. The metabolic pattern on xylose is quite different from that on glucose. The efficiency of xylose assimilation and metabolism in recombinant $\mathrm{Sa}$. cerevisiae is generally much lower than glucose although xylose uptake and utilization has been engineered extensively and improved dramatically (Qi et al., 2015; Hou et al., 2017). Inefficient xylose assimilation leads to carbon starvation-like metabolomic patterns of glycolysis, indicated by the observation of low pools of glycolytic intermediates except for the accumulation of phosphoenolpyruvate, which is required for the production of aromatic compounds through the shikimate pathway (Mert et al., 2017). Growth on glucose induces the expression of hexokinase 2 (Hxk2 encoded by HXK2), activates ethanolproducing metabolism, and represses mitochondrial activities (Moreno and Herrero, 2002). In contrast, this glucose-dependent repression on the respiratory energy metabolism can be dysregulated by xylose (Jin et al., 2004). The mitochondrial genes and the TCA cycle are very active when $\mathrm{Sa}$. cerevisiae are grown on xylose. In addition, xylose can upregulate the glyoxylate pathway and activate cytosolic acetyl-CoA metabolism with enhanced expression of genes encoding aldehyde dehydrogenases and acetyl-CoA synthetase 1 (ALD2, ALD3, $A L D 6$, and ACS1). Meantime, the gene encoding ethanol reoxidizing alcohol dehydrogenase $(\mathrm{ADH} 2)$ is also highly induced upon xylose utilization (Matsushika et al., 2014). Besides, the non-oxidative PPP is activated when recombinant Sa. cerevisiae is grown on xylose, leading to the accumulation of some important intermediates such as E-4-P, which is one of the precursors for the synthesis of aromatic compounds (Figure 2). All of these metabolic properties make $S a$. cerevisiae beneficial for the biosynthesis of many classes of natural products, such as terpenoids, flavonoids, and other polyphenols.

To direct the production of natural compounds from xylose, the relative pathways need to be constructed and introduced into xylose-utilizing Sa. cerevisiae. Currently, production of vitamin A, protopanaxadiol, $p$-coumaric acid, carotenoid, and other natural products has been achieved in Sa. cerevisiae by fermentation on xylose (Table 1; Kwak et al., 2017; Borja et al., 2019; Su et al., 2020). For instance, a lycopene biosynthetic pathway consisting of $C r t E, C r t B$, and $C r t I$ was introduced into xylose-fermenting $S a$. cerevisiae overexpressing native $X K$ and Sc. stipitis-derived XYL1 and XYL2. The PK pathway consisting of xylulose-5-phosphate phosphoketolase (xPK) and phosphotransacetylase (PTA) was further introduced to directly convert xylulose-5-phosphate into acetyl-CoA. The resultant strain produced 1.6-fold more lycopene using the mixture of glucose and xylose than using glucose alone (Su et al., 2020). In another case, squalene-producing recombinant $\mathrm{Sa}$. cerevisiae showed 8-fold higher production on xylose than on glucose (Kwak et al., 2017). A recombinant strain of xylose-metabolizing Sa. cerevisiae was engineered to carry the pathway for $p$-coumaric acid production through the expression of tyrosine ammonia lyase (TAL) and overexpression of some tyrosine biosynthetic pathway genes (Borja et al., 2019). This strain produced $242 \mathrm{mg} / \mathrm{L}$ of $p$-coumaric acid from xylose while the titer was only $5.35 \mathrm{mg} / \mathrm{L}$ on glucose. Moreover, a xylose-fermenting strain 


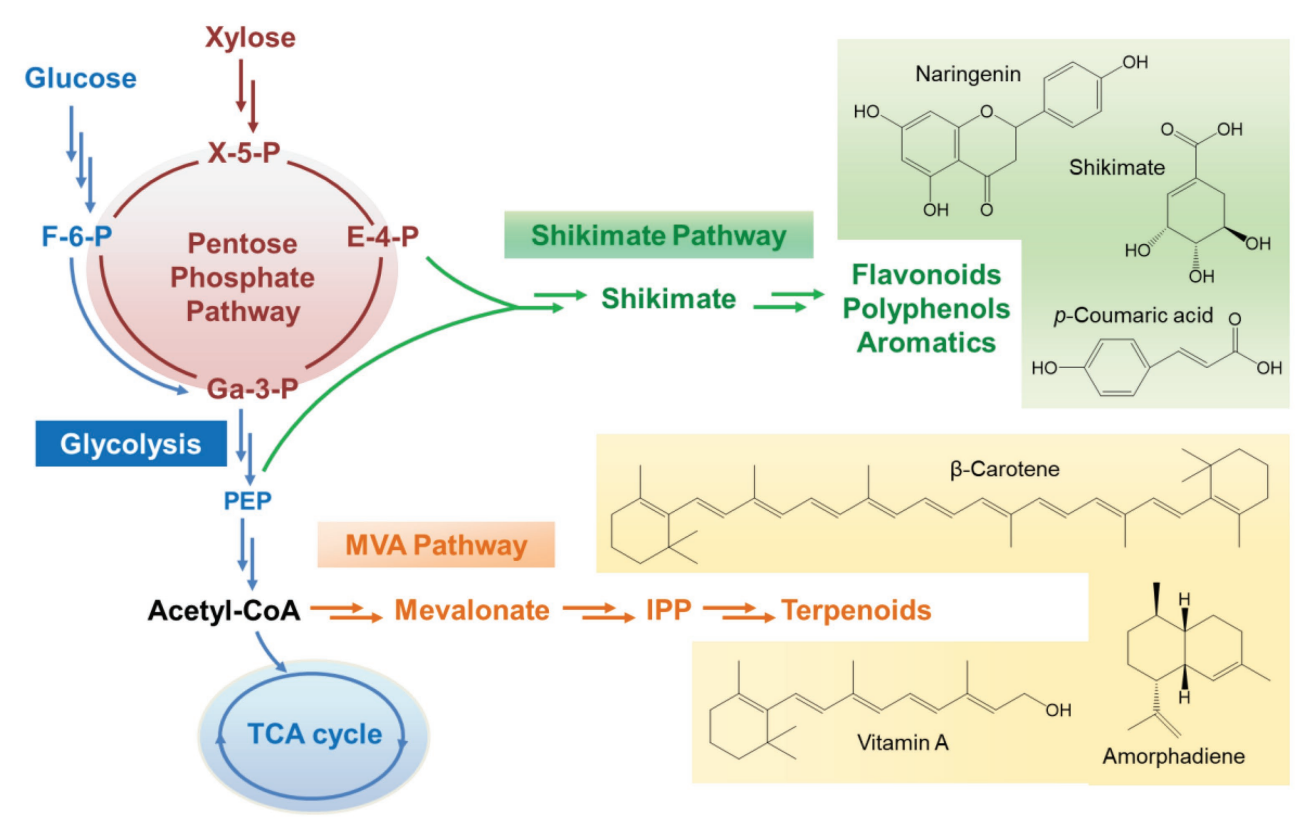

FIGURE 2 | The metabolic flux channel to direct the production of natural products through the shikimate pathway and mevalonate pathway when xylose is employed as the carbon source. X-5-P, xylulose-5-phosphate; E-4-P, erythrose-4-phosphate; F-6-P, fructose-6-phosphate; Ga-3-P, glyceraldehyde-3-phosphate; PEP, phosphoenolpyruvate; IPP, isopentenyl diphosphate.

expressing the biosynthetic pathway of shinorine, a natural sunscreen material, produced a trace amount of shinorine in glucose, whereas the titer was dramatically increased by adding xylose in the medium (Park et al., 2019). This interesting result was related to enhanced PPP flux triggered by xylose and abundant supply of sedoheptulose-7-phosphate, which is the preliminary precursor for the synthesis of shinorine.

\section{BIOSYNTHESIS OF NATURAL PRODUCTS FROM XYLOSE BY YARROWIA LIPOLYTICA}

Yarrowia lipolytica is an obligate aerobe that has a high flux of the TCA cycle and high translational efficiency of mitochondrial genes involved in aerobic respiration (Man and Pilpel, 2007; Christen and Sauer, 2011; Zhu and Jackson, 2015; Abdel-Mawgoud et al., 2018; Shi et al., 2018; Ma et al., 2019). This yeast grows at temperatures below $34^{\circ} \mathrm{C}$ and over a wide $\mathrm{pH}$ range, with metabolic performances varying with cultivation conditions (Egermeier et al., 2017; Abdel-Mawgoud et al., 2018). It is a GRAS microbe due to its low tendency of growing at human body temperature and the low probability of causing only mild infections in immunocompromised people (Groenewald et al., 2014). Phylogenetically dissimilar to other members in the yeast family, Y. lipolytica is considered to be nonconventional (Dujon et al., 2004). Its genome is naturally in haploid form facilitating genetic manipulation, although diploids are occasionally observed (Knutsen et al., 2007; Abdel-Mawgoud et al., 2018). The gene density in this microbe is much lower compared with Sa. cerevisiae, with the large and abundant intergenic regions suitable for gene integration (Dujon et al., 2004; Abdel-Mawgoud et al., 2018; Holkenbrink et al., 2018). The genes in Y. lipolytica are rich in introns (Stajich et al., 2007; Mekouar et al., 2010), the presence of which can positively affect expression levels of the relative exons (Le Hir et al., 2003; Hong et al., 2012; Tai and Stephanopoulos, 2013; Shaul, 2017). In addition, this strain has a broad spectrum of carbon sources, including sugars, acetate, fatty acids, alcohols, waste cooking oil, and so on (Abdel-Mawgoud et al., 2018).

Genetic engineering of Y. lipolytica is generally challenging compared with $\mathrm{Sa}$. cerevisiae. First, it is not easy to precisely integrate gene fragments into the genome of Y. lipolytica at specified loci because this organism prefers NHEJ (Richard et al., 2005), whereas homologous recombination usually occurs only when long homologous arms $(>1 \mathrm{~kb})$ are used (Verbeke et al., 2013). This is attributed to the $\mathrm{Ku} 70 / \mathrm{Ku} 80$ protein heterodimer that repairs breaks in DNA double strands (Lustig, 1999). Deletion of the relevant genes results in much higher frequency in the occurrence of homologous recombination with short (50 bp) homologous arms (Verbeke et al., 2013), and repression of both KU70 and KU80 by CRISPR-dCas9 greatly improves the efficiency of homologous recombination $(>90 \%$; Schwartz et al., 2017a). Second, there are not many engineering tools available for Y. lipolytica given that this yeast has only been used in metabolic engineering for less than two decades. The emergence of Y. lipolytica-based CRISPR systems (Gao et al., 2016; Schwartz et al., 2016, 2017b, 2018; Morse et al., 2018; Zhang et al., 2018), transposon systems (Casaregola et al., 2000; Patterson et al., 2018; Wagner et al., 2018; Yu et al., 2018) 
TABLE 1 | Summary of the biosynthesis of natural products from xylose using yeast strains.

\begin{tabular}{|c|c|c|c|c|}
\hline Chassis & Engineering strategy & Product & Titer (mg/L) & References \\
\hline Scheffersomyces stipitis & $\begin{array}{l}\uparrow D A H P \text { synthase variant } \\
\text { (aro4K220L), } \uparrow \text { aro1(D900A), and } \\
\uparrow \text { Tkt1 }\end{array}$ & Shikimate & 3,110 & Gao et al., 2017 \\
\hline Saccharomyces cerevisiae & 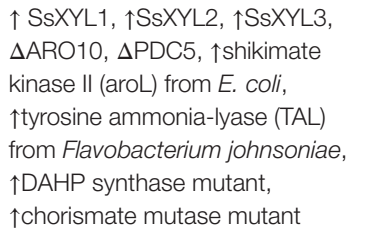 & $p$-coumaric acid & 242 & Borja et al., 2019 \\
\hline Saccharomyces cerevisiae & $\begin{array}{l}\uparrow \mathrm{SsXYL1}(\mathrm{K} 271 \mathrm{~N}), \uparrow \mathrm{SsXY2}, \\
\uparrow \mathrm{XKS}, \uparrow \mathrm{Gal} 2(\mathrm{~N} 376 \mathrm{~F}), \uparrow \mathrm{XPK}, \uparrow \mathrm{PTA}, \\
\uparrow \mathrm{HMG} 1, \uparrow \mathrm{CrtEBI}, \Delta \text { pho13, and } \\
\Delta \text { Ald6 }\end{array}$ & $\beta$-Carotene & 903 & Su et al., 2020 \\
\hline Saccharomyces cerevisiae & $\begin{array}{l}\uparrow \text { SsXYL1, } \text { SsXYL2, } \text { SsXYL3, } \\
\uparrow T K L 1, \uparrow N p R 5600, \uparrow N p R 5599, \\
\uparrow N p R 5598, \uparrow N p R 5597, \text { and } \\
\Delta \text { TAL1 }\end{array}$ & Shinorine & 31 & Park et al., 2019 \\
\hline Saccharomyces cerevisiae & $\begin{array}{l}\uparrow \text { SsXYL1, } \uparrow S s X Y L 2, \uparrow S s X Y L 3 \\
\uparrow T K L 1, \uparrow E R G 10, \uparrow t H M G 1, \uparrow T A L 1, \\
\Delta T A L 1, \Delta \text { pho13, and } \Delta \text { Ald6 }\end{array}$ & Squalene & 532 & Kwak et al., 2017 \\
\hline Saccharomyces cerevisiae & $\begin{array}{l}\uparrow \text { SsXYL1, } \uparrow S s X Y L 2, \uparrow S s X Y L 3, \\
\uparrow T K L 1, \uparrow E R G 10, \uparrow t H M G 1, \uparrow T A L 1, \\
\uparrow A D S, \Delta T A L 1, \Delta \text { pho13, and } \\
\Delta \text { Ald6 }\end{array}$ & Amorphadinene & 254 & Kwak et al., 2017 \\
\hline Saccharomyces cerevisiae & $\begin{array}{l}\uparrow \text { SsXYL1, } \uparrow \text { SsXYL2, } \uparrow S s X Y L 3, \\
\uparrow C r t E / I / Y B, \uparrow B l h, \Delta \text { pho13, and } \\
\Delta \text { Ald6 }\end{array}$ & Vitamin A & 3,350 & Sun et al., 2019 \\
\hline Yarrowia lipolytica & $\begin{array}{l}\uparrow X Y L 1, \uparrow X Y L 2, \uparrow X K S, \uparrow T A L \\
\text { (tyrosine ammonia lyase), } \uparrow 4 C L \text {, } \\
\uparrow C H S, \uparrow C H I \text {, and } \uparrow X T \text { (xylose } \\
\text { transporter, YALIOB00396) }\end{array}$ & Naringenin & 715 & Wei et al., 2020b \\
\hline
\end{tabular}

and artificial genomes (Guo et al., 2020) has greatly facilitated genetic modifications, although delicate design is always required. A detailed description of all the genetic tools and strategies suitable for Y. lipolytica engineering can be found in a very recent review (Ma et al., 2020).

It had long been thought that Y. lipolytica could not naturally utilize xylose as the only carbon source (Blazeck et al., 2014; Zhao et al., 2015), and early attempts enabling xylose metabolism relied on introduction of heterologous pathways from S. stipitis, although such a phenotype tends to be unstable and needs to be strengthened through further adaptation for higher expression levels of XR-encoding genes (Ledesma-Amaro et al., 2016; Wu et al., 2019). However, recent studies have discovered a functional endogenous xylose-metabolizing pathway, which can be highly efficient after facile engineering. Wildtype strain PO1f (ATCC MYA-2613) carries genes encoding active XR, XDH, and XKS at low expression levels, which can be upregulated when xylose is used as the sole carbon source (Ryu et al., 2015; Rodriguez et al., 2016), although it is reported that the two XR-encoding genes are constitutively expressed at stable levels irrespective of the growth stage or the carbon source used for cell cultivation (Rodriguez et al., 2016). Overexpression of XDH- or XKS-encoding gene alone or in combination considerably improves xylose assimilation and conversion (Ryu et al., 2015; Rodriguez et al., 2016), whereas simultaneous overexpression of XR and XKS does not enable cells to grow on xylose (Wu et al., 2019), suggesting expression of XDH and XKS is the bottleneck in natural xylose metabolism (Ryu et al., 2015; Rodriguez et al., 2016). In addition, strain PO1f contains five putative xylose-specific transporters (Ryu et al., 2015), and overexpression of the transporter YALI0B00396 (a co-transporter for xylose and cellobiose) improves xylose uptake (Ryu and Trinh, 2018; Wu et al., 2019; Wei et al., 2020b). Interestingly, xylose metabolism in strain PO1f is not repressed by the presence of glucose (Ryu et al., 2015) as long as glucose concentration is below $2 \mathrm{~g} / \mathrm{L}$ (Rodriguez et al., 2016), which is distinct from another natural xylose-utilizing strain PO1g whose xylose metabolism is mildly repressed by glucose (Tsigie et al., 2011). These studies have opened up an avenue to xylose utilization in $Y$. lipolytica without the need of complicated genetic engineering, despite the fact that xylose-based cell growth is, in many cases, slower compared with cell growth on glucose.

Yarrowia lipolytica has excellent capability of accumulating acetyl-CoA and malonyl-CoA, and is hence a theoretically preferred host for the production of fatty acids, terpenoids, flavonoids, and other compounds that use acetyl/malonyl-CoA as a precursor (Abdel-Mawgoud et al., 2018). In the past few years, the potential of this host has been greatly explored in the biosynthesis of useful compounds such as lipids and biofuels from various carbon sources (Zhu and Jackson, 2015; 
Du et al., 2016; Ledesma-Amaro et al., 2016; Lv et al., 2019; Ma et al., 2019; Palmer et al., 2020; Shang et al., 2020); however, xylose-based biosynthesis of natural products has been scarcely investigated despite attempts to understand and improve xylose metabolism in Y. lipolytica (Tsigie et al., 2011; Ryu et al., 2015; Rodriguez et al., 2016; Wei et al., 2020b). On one hand, this is attributed to the lower growth rate and biomass accumulation when cells are cultivated in xylose than in glucose (LedesmaAmaro et al., 2016); on the other hand, the functional and highly efficient expression of heterologous metabolic pathways relevant to natural product biosynthesis depends on sophisticated tools for genetic manipulation, which are still very limited. Recently, strain ATCC 201249 was engineered to produce the isoprenoid compound protopanaxadiol from xylose (Wu et al., 2019). Overexpression of XR (with K270R/N272D mutations to convert cofactor preference from NADPH to NADH) and $\mathrm{XDH}$ from Sc. stipitis and endogenous XKS, followed by xylose adaptation and overexpression of xylose transporter YALI0B00396, enabled the strain to consume $20 \mathrm{~g} / \mathrm{L}$ of xylose in $72 \mathrm{~h}$, reaching an $\mathrm{OD}_{600}$ of 32 in shake flasks. Introduction of the biosynthetic pathway of protopanaxadiol together with fusion expression of pathway enzymes and overexpression of genes involved in precursor supply resulted in a titer of $300 \mathrm{mg} / \mathrm{L}$ using fed-batch fermentation. In this process, xylose was preferred to glucose as the carbon source, due to the fact that glucose supported fast cell growth and led to rapid generation and accumulation of acetyl-CoA, which was then channeled for the generation of metabolites other than precursors (Wu et al., 2019). In the latest study, a xylose-inducible machinery was designed to couple endogenous xylose utilization and naringenin biosynthesis, producing $511 \mathrm{mg} / \mathrm{L}$ of naringenin in $120 \mathrm{~h}$ from $20 \mathrm{~g} / \mathrm{L}$ glucose and $20 \mathrm{~g} / \mathrm{L}$ xylose in shake flasks without externally added precursors; the titer was further increased to $715 \mathrm{mg} / \mathrm{L}$ in $144 \mathrm{~h}$ with an elevated xylose concentration (60 g/L; Wei et al., 2020a,b).

Recently, Y. lipolytica has been engineered to produce triglycerides, which can form oil droplets inside cells. These droplets are capable of trapping and storing certain heterologously generated compounds, whose overproduction imposes a threat to cell growth. Thus, Y. lipolytica can be used as a good host to produce hydrophobic natural products, including terpenoids and aromatics, which are prone to formation of crystals in the cytosol that are harmful to the host. Concomitant generation of triglycerides allows hydrophobic natural compounds to be stored in oil droplets, and hence protects the host and guarantees continuous production of the target compounds.

\section{CONCLUDING REMARKS}

Biorefinery of lignocellulosic hydrolysates as feedstocks to produce advanced fuels and chemicals holds great promise to develop sustainable bioeconomy. As the second most abundant monosaccharide in lignocellulosic hydrolysates, xylose has been attracting much attention for its efficient utilization and conversion into valuable compounds beyond ethanol. Metabolic engineering of yeasts to convert xylose into natural products is a promising option to implement the biotransformation due to some special characteristics of certain yeast strains, such as moderate tolerance to toxic inhibitors in lignocellulosic hydrolysates, and moderate compatibility of heterologous pathway genes derived from both prokaryotes and eukaryotes. Recently, metabolic engineering of Sc. stipitis, Sa. cerevisiae and Y. lipolytica has been accomplished for the biosynthesis of natural products, mainly terpenoids, flavonoids, and aromatics. Some of these microbial production processes function better on xylose than on glucose, as xylose induces reprograming of central metabolism and enhances the supply of key precursors, such as acetyl-CoA.

Currently, most of the studies are centered on Sa. cerevisiae due to the availability of facile genetic engineering tools and methods. However, this yeast relies on heterologous pathways for xylose utilization, which are not always highly efficient due to cofactor imbalance. Sc. stipitis is a superior xyloseutilizer and, when grown on xylose, generates many intermediate metabolites that are critical for the formation of natural compounds. Nevertheless, the lack of physiological information and genetic engineering tools limits its applications mainly to ethanol production. It appears that Y. lipolytica has the merits of both Sa. cerevisiae and Sc. stipitis. This organism contains native xylose-utilizing genes, and can be turned into an efficient xylose-utilizing factory upon overexpression of these native genes. In addition, the gene arrangement in its genome as well as the established gene manipulation strategies allows for the construction of $Y$. lipolytica workhorse for the generation of natural compounds from xylose. However, the growth rate of this yeast is generally much lower on xylose than on glucose, making the whole bioconversion inefficient. Extensive engineering work is needed to overcome these difficulties.

Another challenge associated with the utilization of lignocellulosic hydrolysates is the presence of toxic compounds, such as furfural and phenolics, generated in the physiochemical pretreatment of biomass. These compounds greatly suppress the growth of fermentation microorganisms and inhibit the biosynthesis of the target products. Thus, development of highly tolerant yeast strains is of critical significance. Strain adaptation or engineering should be considered for the construction of highly efficient yeast cell factories capable of utilizing lignocellulosic hydrolysates for the generation of natural products.

\section{AUTHOR CONTRIBUTIONS}

JZ, WQ, and XW conceived the project. JZ, MY, and XW wrote the manuscript. All the authors read and approved the manuscript.

\section{FUNDING}

The authors are grateful to the financial support from National Natural Science Foundation of China (31900114), and the Natural Science Foundation of Shaanxi Province (2020JQ-702 and 2020JQ-703). This work was also supported by the startup funds from Shaanxi University of Science and Technology awarded to JZ and XW. 


\section{REFERENCES}

Abdel-Mawgoud, A. M., Markham, K. A., Palmer, C. M., Liu, N., Stephanopoulos, G., and Alper, H. S. (2018). Metabolic engineering in the host Yarrowia lipolytica. Metab. Eng. 50, 192-208. doi: 10.1016/j. ymben.2018.07.016

Blazeck, J., Hill, A., Liu, L., Knight, R., Miller, J., Pan, A., et al. (2014). Harnessing Yarrowia lipolytica lipogenesis to create a platform for lipid and biofuel production. Nat. Commun. 5:3131. doi: 10.1038/ncomms4131

Borgström, C., Wasserstrom, L., Almqvist, H., Broberg, K., Klein, B., Noack, S., et al. (2019). Identification of modifications procuring growth on xylose in recombinant Saccharomyces cerevisiae strains carrying the Weimberg pathway. Metab. Eng. 55, 1-11. doi: 10.1016/j.ymben.2019.05.010

Borja, G. M., Rodriguez, A., Campbell, K., Borodina, I., Chen, Y., and Nielsen, J. (2019). Metabolic engineering and transcriptomic analysis of Saccharomyces cerevisiae producing $p$-coumaric acid from xylose. Microb. Cell Factories 18:191. doi: 10.1186/s12934-019-1244-4

Bruinenberg, P. M., de Bot, P. H. M., van Dijken, J. P., and Scheffers, W. A. (1984). NADH-linked aldose reductase: the key to anaerobic alcoholic fermentation of xylose by yeasts. Appl. Microbiol. Biotechnol. 19, 256-260. doi: $10.1007 / \mathrm{BF} 00251847$

Cao, M., Gao, M., Lopez-Garcia, C. L., Wu, Y., Seetharam, A. S., Severin, A. J., et al. (2017). Centromeric DNA facilitates nonconventional yeast genetic engineering. ACS Synth. Biol. 6, 1545-1553. doi: 10.1021/acssynbio.7b00046

Cao, M., Gao, M., Ploessl, D., Song, C., and Shao, Z. (2018). CRISPR-mediated genome editing and gene repression in Scheffersomyces stipitis. Biotechnol. J. 13:e1700598. doi: 10.1002/biot.201700598

Casaregola, S., Neuvéglise, C., Lépingle, A., Bon, E., Feynerol, C., Artiguenave, F., et al. (2000). Genomic exploration of the Hemiascomycetous yeasts: 17 . Yarrowia lipolytica. FEBS Lett. 487, 95-100. doi: 10.1016/s0014-5793(00)02288-2

Christen, S., and Sauer, U. (2011). Intracellular characterization of aerobic glucose metabolism in seven yeast species by ${ }^{13} \mathrm{C}$ flux analysis and metabolomics. FEMS Yeast Res. 11, 263-272. doi: 10.1111/j.1567-1364.2010.00713.x

Does, A. L., and Bisson, L. F. (1989). Characterization of xylose uptake in the yeasts Pichia heedii and Pichia stipitis. Appl. Environ. Microbiol. 55, 159-164. doi: 10.1128/AEM.55.1.159-164.1989

Du, H. X., Xiao, W. H., Wang, Y., Zhou, X., Zhang, Y., and Liu, D., et al. (2016). Engineering Yarrowia lipolytica for campesterol overproduction. PLoS One. 11:e0146773. doi: 10.1371/journal.pone.0146773

Dujon, B., Sherman, D., Fischer, G., Durrens, P., Casaregola, S., Lafontaine, I., et al. (2004). Genome evolution in yeasts. Nature. 430, 35-44. doi: 10.1038/ nature 02579

Egermeier, M., Russmayer, H., Sauer, M., and Marx, H. (2017). Metabolic flexibility of Yarrowia lipolytica growing on glycerol. Front. Microbiol. 8:49. doi: $10.3389 /$ fmicb.2017.00049

Feng, X., and Zhao, H. (2013). Investigating glucose and xylose metabolism in Saccharomyces cerevisiae and Scheffersomyces stipitis via ${ }^{13} \mathrm{C}$ metabolic flux analysis. AICHE J. 59, 3195-3202. doi: 10.1002/aic.14182

Gao, M., Cao, M., Suástegui, M., Walker, J., Rodriguez Quiroz, N., Wu, Y., et al. (2017). Innovating a nonconventional yeast platform for producing Shikimate as the building block of high-value aromatics. ACS Synth. Biol. 6, 29-38. doi: 10.1021/acssynbio.6b00132

Gao, S., Tong, Y., Wen, Z., Zhu, L., Ge, M., Chen, D., et al. (2016). Multiplex gene editing of the Yarrowia lipolytica genome using the CRISPR-Cas9 system. J. Ind. Microbiol. Biotechnol. 43, 1085-1093. doi: 10.1007/ s10295-016-1789-8

Groenewald, M., Boekhout, T., Neuvéglise, C., and Gaillardin, C., van Dijck, P. W., and Wyss, M. (2014). Yarrowia lipolytica: safety assessment of an oleaginous yeast with a great industrial potential. Crit. Rev. Microbiol. 40, 187-206. doi: 10.3109/1040841X.2013.770386

Guo, Z. P., Borsenberger, V., Croux, C., Duquesne, S., Truan, G., Marty, A., et al. (2020). An artificial chromosome ylAC enables efficient assembly of multiple genes in Yarrowia lipolytica for biomanufacturing. Commun. Biol. 3:199. doi: 10.1038/s42003-020-0936-y

Holkenbrink, C., Dam, M. I., Kildegaard, K. R., Beder, J., Dahlin, J., Doménech Belda, D., et al. (2018). EasyCloneYALI: CRISPR/Cas9-based synthetic toolbox for engineering of the yeast Yarrowia lipolytica. Biotechnol. J. 13:1700543. doi: 10.1002/biot.201700543
Hong, S. -P., Seip, J., Walters-Pollak, D., Rupert, R., Jackson, R., Xue, Z., et al. (2012). Engineering Yarrowia lipolytica to express secretory invertase with strong FBA1IN promoter. Yeast. 29, 59-72. doi: 10.1002/yea.1917

Hou, J., Qiu, C., Shen, Y., Li, H., and Bao, X. (2017). Engineering of Saccharomyces cerevisiae for the efficient co-utilization of glucose and xylose. FEMS Yeast Res. 17:fox034. doi: 10.1093/femsyr/fox034

Jagtap, S. S., and Rao, C. V. (2018). Microbial conversion of xylose into useful bioproducts. Appl. Microbiol. Biotechnol. 102, 9015-9036. doi: 10.1007/ s00253-018-9294-9

Jeffries, T. W., Grigoriev, I. V., Grimwood, J., Laplaza, J. M., Aerts, A., Salamov, A., et al. (2007). Genome sequence of the lignocellulose-bioconverting and xylose-fermenting yeast Pichia stipitis. Nat. Biotechnol. 25, 319-326. doi: $10.1038 /$ nbt 1290

Jeppsson, H., Alexander, N. J., and Hahn-Hagerdal, B. (1995). Existence of cyanide-insensitive respiration in the yeast Pichia stipitis and its possible influence on product formation during xylose utilization. Appl. Environ. Microbiol. 61, 2596-2600. doi: 10.1128/AEM.61.7.2596-2600.1995

Jin, Y. -S., Laplaza, J. M., and Jeffries, T. W. (2004). Saccharomyces cerevisiae engineered for xylose metabolism exhibits a respiratory response. Appl. Environ. Microbiol. 70, 6816-6825. doi: 10.1128/AEM.70.11.6816-6825.2004

Kilian, S. G., and van Uden, N. (1988). Transport of xylose and glucose in the xylose-fermenting yeast Pichia stipitis. Appl. Microbiol. Biotechnol. 27, 545-548. doi: 10.1007/BF00451629

Kim, S. R., Park, Y. -C., Jin, Y. -S., and Seo, J. -H. (2013). Strain engineering of Saccharomyces cerevisiae for enhanced xylose metabolism. Biotechnol. Adv. 31, 851-861. doi: 10.1016/j.biotechadv.2013.03.004

Knutsen, A. K., Robert, V., Poot, G. A., Epping, W., Figge, M., and Holst-Jensen, A., et al. (2007). Polyphasic re-examination of Yarrowia lipolytica strains and the description of three novel candida species: Candida oslonensis sp. nov., Candida alimentaria sp. nov. and Candida hollandica sp. nov. Int. J. Syst. Evol. Microbiol. 57, 2426-2435. doi: 10.1099/ijs.0.65200-0

Konishi, J., Fukuda, A., Mutaguchi, K., and Uemura, T. (2015). Xylose fermentation by Saccharomyces cerevisiae using endogenous xylose-assimilating genes. Biotechnol. Lett. 37, 1623-1630. doi: 10.1007/s10529-015-1840-2

Kuyper, M., Harhangi, H. R., Stave, A. K., Winkler, A. A., Jetten, M. S. M., de Laat, W. T. A. M., et al. (2003). High-level functional expression of a fungal xylose isomerase: the key to efficient ethanolic fermentation of xylose by Saccharomyces cerevisiae? FEMS Yeast Res. 4, 69-78. doi: 10.1016/ S1567-1356(03)00141-7

Kwak, S., and Jin, Y. -S. (2017). Production of fuels and chemicals from xylose by engineered Saccharomyces cerevisiae: a review and perspective. Microb. Cell Factories 16:82. doi: 10.1186/s12934-017-0694-9

Kwak, S., Jo, J. H., Yun, E. J., Jin, Y. -S., and Seo, J. -H. (2019). Production of biofuels and chemicals from xylose using native and engineered yeast strains. Biotechnol. Adv. 37, 271-283. doi: 10.1016/j.biotechadv.2018.12.003

Kwak, S., Kim, S. R., Xu, H., Zhang, G. C., Lane, S., Kim, H., et al. (2017). Enhanced isoprenoid production from xylose by engineered Saccharomyces cerevisiae. Biotechnol. Bioeng. 114, 2581-2591. doi: 10.1002/bit.26369

Le Hir, H., Nott, A., and Moore, M. J. (2003). How introns influence and enhance eukaryotic gene expression. Trends Biochem. Sci. 28, 215-220. doi: 10.1016/S0968-0004(03)00052-5

Ledesma-Amaro, R., Lazar, Z., Rakicka, M., Guo, Z., Fouchard, F., Coq, A. -M. C. -L., et al. (2016). Metabolic engineering of Yarrowia lipolytica to produce chemicals and fuels from xylose. Metab. Eng. 38, 115-124. doi: 10.1016/j. ymben.2016.07.001

Li, X., Chen, Y., and Nielsen, J. (2019). Harnessing xylose pathways for biofuels production. Curr. Opin. Biotechnol. 57, 56-65. doi: 10.1016/j.copbio.2019.01.006

Ligthelm, M. E., and Prior, B. A., du Preez, J. C., and Brandt, V. (1988). An investigation of $\mathrm{D}-\left\{1-{ }^{13} \mathrm{C}\right\}$ xylose metabolism in Pichia stipitis under aerobic and anaerobic conditions. Appl. Microbiol. Biotechnol. 28, 293-296. doi:10.1007/ BF00250458

Lustig, A. J. (1999). The Kudos of non-homologous end-joining. Nat. Genet. 23, 130-131. doi: 10.1038/13755

Lv, Y., Marsafari, M., Koffas, M., Zhou, J., and Xu, P. (2019). Optimizing oleaginous yeast cell factories for flavonoids and hydroxylated flavonoids biosynthesis. ACS Synth. Biol. 8, 2514-2523. doi: 10.1021/acssynbio.9b00193

Ma, J., Gu, Y., Marsafari, M., and Xu, P. (2020). Synthetic biology, systems biology, and metabolic engineering of Yarrowia lipolytica toward a sustainable 
biorefinery platform. J. Ind. Microbiol. Biotechnol. 47, 845-862. doi: 10.1007/ s10295-020-02290-8

Ma, Y. -R., Wang, K. -F., Wang, W. -J., Ding, Y., Shi, T. -Q., Huang, H., et al. (2019). Advances in the metabolic engineering of Yarrowia lipolytica for the production of terpenoids. Bioresour. Technol. 281, 449-456. doi: 10.1016/j. biortech.2019.02.116

Man, O., and Pilpel, Y. (2007). Differential translation efficiency of orthologous genes is involved in phenotypic divergence of yeast species. Nat. Genet. 39, 415-421. doi: $10.1038 / \mathrm{ng} 1967$

Matsushika, A., Goshima, T., and Hoshino, T. (2014). Transcription analysis of recombinant industrial and laboratory Saccharomyces cerevisiae strains reveals the molecular basis for fermentation of glucose and xylose. Microb. Cell Factories 13:16. doi: 10.1186/1475-2859-13-16

Mekouar, M., Blanc-Lenfle, I., Ozanne, C., Da Silva, C., Cruaud, C., and Wincker, P., et al. (2010). Detection and analysis of alternative splicing in Yarrowia lipolytica reveal structural constraints facilitating nonsense-mediated decay of intron-retaining transcripts. Genome Biol. 11:R65. doi: 10.1186/ gb-2010-11-6-r65

Mert, M. J., Rose, S. H., la Grange, D. C., Bamba, T., Hasunuma, T., Kondo, A., et al. (2017). Quantitative metabolomics of a xylose-utilizing Saccharomyces cerevisiae strain expressing the Bacteroides thetaiotaomicron xylose isomerase on glucose and xylose. J. Ind. Microbiol. Biotechnol. 44, 1459-1470. doi: $10.1007 / \mathrm{s} 10295-017-1969-1$

Moreno, F., and Herrero, P. (2002). The hexokinase 2-dependent glucose signal transduction pathway of Saccharomyces cerevisiae. FEMS Microbiol. Rev. 26, 83-90. doi: 10.1111/j.1574-6976.2002.tb00600.x

Morse, N. J., Wagner, J. M., Reed, K. B., Gopal, M. R., Lauffer, L. H., and Alper, H. S. (2018). T7 polymerase expression of guide RNAs in vivo allows exportable CRISPR-Cas9 editing in multiple yeast hosts. ACS Synth. Biol. 7, 1075-1084. doi: 10.1021/acssynbio.7b00461

Palmer, C. M., Miller, K. K., Nguyen, A., and Alper, H. S. (2020). Engineering 4-coumaroyl-CoA derived polyketide production in Yarrowia lipolytica through a $\beta$-oxidation mediated strategy. Metab. Eng. 57, 174-181. doi: 10.1016/j. ymben.2019.11.006

Park, S. -H., Lee, K., Jang, J. W., and Hahn, J. -S. (2019). Metabolic engineering of Saccharomyces cerevisiae for production of shinorine, a sunscreen material, from xylose. ACS Synth. Biol. 8, 346-357. doi: 10.1021/ acssynbio. 8 b00388

Patterson, K., Yu, J., Landberg, J., Chang, I., Shavarebi, F., and Bilanchone, V., et al. (2018). Functional genomics for the oleaginous yeast Yarrowia lipolytica. Metab. Eng. 48, 184-196. doi: 10.1016/j.ymben.2018.05.008

Qi, X., Zha, J., Liu, G. -G., Zhang, W., Li, B. -Z., and Yuan, Y. -J. (2015). Heterologous xylose isomerase pathway and evolutionary engineering improve xylose utilization in Saccharomyces cerevisiae. Front. Microbiol. 6:1165. doi: 10.3389/fmicb.2015.01165

Richard, G. F., Kerrest, A., Lafontaine, I., and Dujon, B. (2005). Comparative genomics of hemiascomycete yeasts: genes involved in DNA replication, repair, and recombination. Mol. Biol. Evol. 22, 1011-1023. doi: 10.1093/ $\mathrm{molbev} / \mathrm{msi} 083$

Rodriguez, G. M., Hussain, M. S., Gambill, L., Gao, D., Yaguchi, A., and Blenner, M. (2016). Engineering xylose utilization in Yarrowia lipolytica by understanding its cryptic xylose pathway. Biotechnol. Biofuels 9:149. doi: 10.1186/s13068-016-0562-6

Ryu, S., Hipp, J., and Trinh, C. T. (2015). Activating and elucidating metabolism of complex sugars in Yarrowia lipolytica. Appl. Environ. Microbiol. 82, 1334-1345. doi: 10.1128/AEM.03582-15

Ryu, S., and Trinh, C. T. (2018). Understanding functional roles of native pentose-specific transporters for activating dormant pentose metabolism in Yarrowia lipolytica. Appl. Environ. Microbiol. 84, e02146-e021417. doi: 10.1128/ AEM.02146-17

Schwartz, C., Curtis, N., Löbs, A. -K., and Wheeldon, I. (2018). Multiplexed CRISPR activation of cryptic sugar metabolism enables Yarrowia lipolytica growth on cellobiose. Biotechnol. J. 13:1700584. doi: 10.1002/biot.201700584

Schwartz, C., Frogue, K., Ramesh, A., Misa, J., and Wheeldon, I. (2017a). CRISPRi repression of nonhomologous end-joining for enhanced genome engineering via homologous recombination in Yarrowia lipolytica. Biotechnol. Bioeng. 114, 2896-2906. doi: 10.1002/bit.26404

Schwartz, C. M., Hussain, M. S., Blenner, M., and Wheeldon, I. (2016). Synthetic RNA polymerase III promoters facilitate high-efficiency CRISPR-Cas9-mediated genome editing in Yarrowia lipolytica. ACS Synth. Biol. 5, 356-359. doi: 10.1021/acssynbio.5b00162

Schwartz, C., Shabbir-Hussain, M., Frogue, K., Blenner, M., and Wheeldon, I. (2017b). Standardized markerless gene integration for pathway engineering in Yarrowia lipolytica. ACS Synth. Biol. 6, 402-409. doi: 10.1021/acssynbio.6b00285

Shang, Y., Wei, W., Zhang, P., and Ye, B. -C. (2020). Engineering Yarrowia lipolytica for enhanced production of arbutin. J. Agric. Food Chem. 68, 1364-1372. doi: 10.1021/acs.jafc.9b07151

Shaul, O. (2017). How introns enhance gene expression. Int. J. Biochem. Cell Biol. 91, 145-155. doi: 10.1016/j.biocel.2017.06.016

Shi, N. -Q., Davis, B., Sherman, F., Cruz, J., and Jeffries, T. W. (1999). Disruption of the cytochrome $\mathrm{c}$ gene in xylose-utilizing yeast Pichia stipitis leads to higher ethanol production. Yeast 15, 1021-1030. doi: 10.1002/(SICI)1097 -0061(199908) 15:11<1021::AID-YEA429>3.0.CO;2-V

Shi, T. -Q., Huang, H., Kerkhoven, E. J., and Ji, X. -J. (2018). Advancing metabolic engineering of Yarrowia lipolytica using the CRISPR/Cas system. Appl. Microbiol. Biotechnol. 102, 9541-9548. doi: 10.1007/s00253-018-9366-x

Stajich, J. E., Dietrich, F. S., and Roy, S. W. (2007). Comparative genomic analysis of fungal genomes reveals intron-rich ancestors. Genome Biol. 8:R223. doi: $10.1186 / \mathrm{gb}-2007-8-10-\mathrm{r} 223$

Su, B., Song, D., and Zhu, H. (2020). Metabolic engineering of Saccharomyces cerevisiae for enhanced carotenoid production from xylose-glucose mixtures. Front. Bioeng. Biotechnol. 8:435. doi: 10.3389/fbioe.2020.00435

Sun, L., Kwak, S., and Jin, Y. S. (2019). Vitamin A production by engineered Saccharomyces cerevisiae from xylose via two-phase in situ extraction. ACS Synth. Biol. 8, 2131-2140. doi: 10.1021/acssynbio.9b00217

Tai, M., and Stephanopoulos, G. (2013). Engineering the push and pull of lipid biosynthesis in oleaginous yeast Yarrowia lipolytica for biofuel production. Metab. Eng. 15, 1-9. doi: 10.1016/j.ymben.2012.08.007

Tsigie, Y. A., Wang, C. -Y., Truong, C. -T., and Ju, Y. -H. (2011). Lipid production from Yarrowia lipolytica Po1g grown in sugarcane bagasse hydrolysate. Bioresour. Technol. 102, 9216-9222. doi: 10.1016/j.biortech.2011.06.047

Verbeke, J., Beopoulos, A., and Nicaud, J. -M. (2013). Efficient homologous recombination with short length flanking fragments in Ku70 deficient Yarrowia lipolytica strains. Biotechnol. Lett. 35, 571-576. doi: 10.1007/s10529-012-1107-0

Wagner, J. M., Williams, E. V., and Alper, H. S. (2018). Developing a piggyBac transposon system and compatible selection markers for insertional mutagenesis and genome engineering in Yarrowia lipolytica. Biotechnol. J. 13:1800022. doi: 10.1002/biot.201800022

Wei, W., Shang, Y., Zhang, P., Liu, Y., You, D., Yin, B., et al. (2020a). Engineering prokaryotic transcriptional activator XylR as a xylose-inducible biosensor for transcription activation in yeast. ACS Synth. Biol. 9, 1022-1029. doi: 10.1021/acssynbio.0c00122

Wei, W., Zhang, P., Shang, Y., Zhou, Y., and Ye, B. -C. (2020b). Metabolically engineering of Yarrowia lipolytica for the biosynthesis of naringenin from a mixture of glucose and xylose. Bioresour. Technol. 314:123726. doi: 10.1016/j. biortech.2020.123726

Wu, Y., Xu, S., Gao, X., Li, M., Li, D., and Lu, W. (2019). Enhanced protopanaxadiol production from xylose by engineered Yarrowia lipolytica. Microb. Cell Factories 18:83. doi: 10.1186/s12934-019-1136-7

Yu, J., Landberg, J., Shavarebi, F., Bilanchone, V., Okerlund, A., Wanninayake, U., et al. (2018). Bioengineering triacetic acid lactone production in Yarrowia lipolytica for pogostone synthesis. Biotechnol. Bioeng. 115, 2383-2388. doi: 10.1002/bit.26733

Zha, J., Hu, M. -L., Shen, M. -H., Li, B. -Z., Wang, J. -Y., and Yuan, Y. -J. (2012). Balance of XYL1 and XYL2 expression in different yeast chassis for improved xylose fermentation. Front. Microbiol. 3:355. doi: 10.3389/fmicb.2012.00355

Zha, J., Shen, M., Hu, M., Song, H., and Yuan, Y. (2014). Enhanced expression of genes involved in initial xylose metabolism and the oxidative pentose phosphate pathway in the improved xylose-utilizing Saccharomyces cerevisiae through evolutionary engineering. J. Ind. Microbiol. Biotechnol. 41, 27-39. doi: 10.1007/s10295-013-1350-y

Zhang, J. -L., Peng, Y. -Z., Liu, D., Liu, H., Cao, Y. -X., Li, B. -Z., et al. (2018). Gene repression via multiplex gRNA strategy in Y. lipolytica. Microb. Cell Factories 17:62. doi: 10.1186/s12934-018-0909-8

Zhao, C., Gu, D., Nambou, K., Wei, L., Chen, J., Imanaka, T., et al. (2015). Metabolome analysis and pathway abundance profiling of Yarrowia lipolytica cultivated on different carbon sources. J. Biotechnol. 206, 42-51. doi: 10.1016/j. jbiotec.2015.04.005 
Zhu, Q., and Jackson, E. N. (2015). Metabolic engineering of Yarrowia lipolytica for industrial applications. Curr. Opin. Biotechnol. 36, 65-72. doi: 10.1016/j. copbio.2015.08.010

Conflict of Interest: The authors declare that the research was conducted in the absence of any commercial or financial relationships that could be construed as a potential conflict of interest.
Copyright () 2021 Zha, Yuwen, Qian and Wu. This is an open-access article distributed under the terms of the Creative Commons Attribution License (CC BY). The use, distribution or reproduction in other forums is permitted, provided the original author(s) and the copyright owner(s) are credited and that the original publication in this journal is cited, in accordance with accepted academic practice. No use, distribution or reproduction is permitted which does not comply with these terms. 